\title{
LOS IMPACTOS MULTIPLES DE LAS EMPRESAS ELÉCTRICAS GLOBALES. EL CASO DE IBERDROLA EN MÉXICO
}

\author{
MULTIPLE IMPACTS OF GLOBAL ELECTRIC \\ COMPANIES. THE CASE OF IBERDROLA IN MEXICO.
}

\author{
Luis Miguel Uharte Pozas \\ Universidad del País Vasco/Euskal Herriko Unibertsitatea (UPV/EHU) \\ Facultad de Filosofía. Avenida Tolosa, 70. 20018 \\ luismiguel.uharte@ehu.es
}

Resumen: Existe abundante investigación antecedente sobre los impactos sociales negativos de los proyectos de empresas globales en regiones en desarrollo. Los impactos negativos más comúnmente identificados son: la pérdida de soberanía; su mercantilización de los servicios públicos privatizados; la práctica del lobby; la falta de transparencia, la corrupción, la criminalización social, la precarización laboral y la vulneración de derechos de los pueblos indígenas. El objetivo de este trabajo es evaluar los posibles impactos sociales negativos de los proyectos en México de la compañía eléctrica española Iberdrola. Por lo tanto se concentra en conocer la posición de los sectores críticos con la empresa en México, y analizar sus argumentos y posiciones. La metodología aplicada es de evaluación. Se sustenta en la recopilación de documentación de diversas fuentes (instituciones públicas y empresariales, investigadores, movimientos sociales), y en las entrevistas realizadas a diferentes agentes (afectados, académicos, técnicos...). Los resultados destacan la buena acogida de la empresa por parte de los poderes políticos locales y centrales; frente al descontento popular que culpa a las multinacionales por la continuada subida del precio de la energía eléctrica. El cumplimiento del código ético de la empresa en todos los territorios en que opera es otra cuestión que se discute.

Palabras clave: empresas globales, electricidad, impacto negativo, critica, código ético, opinión pública, vulneración de derechos, lobby, energiía eólica.
Abstract: Abundant research exists on negative social impacts of projects located by global companies in developing regions. The most commonly identified negative impacts are: loss of sovereignty; commercialization of privatized public services; the practice of lobbying; the lack of transparency; corruption; social criminalization; job insecurity; and violation of rights of indigenous peoples. The aim of this study is to evaluate the possible negative social impacts of projects of the Spanish power company Iberdrola in Mexico. Therefore, it focuses on knowing the positions of the critical sectors of the company operating in Mexico, and analyzing the company's arguments and positions. The applied methodology is assessment. It relies on the collection of documents from various sources (public and business institutions, researchers, social movements), and interviews with different agents (affected individuals, academics, technicians). The results contrast the favorable reception of the company by local and central political powers with discontent by the public, which blames multinationals for the continued rise in the price of electricity. Compliance with the company's code of ethics in all territories in which it operates is another issue that is discussed.

Keywords: global companies, electricity, negative impact, critical, ethical code, public opinion, violation of rights, lobbying, wind energy 


\section{Introducción}

Este texto tiene como objetivo fundamental evaluar los posibles impactos que la empresa global española Iberdrola está provocando en México, desde que se instaló en el país en la década del noventa. La metodología se sustenta en la recopilación de documentación de diversas fuentes (organismos públicos, instituciones empresariales, investigadores, movimientos sociales) y en las entrevistas que realizamos durante el mes de marzo de 2012 a diferentes agentes (afectados, académicos, técnicos...), tanto en la capital del país, como en el Estado de Oaxaca, donde Iberdrola tiene importantes negocios eólicos.

En el primer capítulo se realiza una descripción general de la evolución del sector eléctrico en México y del proceso de privatización abierto en los años noventa, además de identificar los principales proyectos que Iberdrola tiene en el país. El segundo apartado tiene un carácter más teórico y se ocupa de enumerar los diversos impactos que generan en diferentes ámbitos (político, económico, social, ambiental, cultural...), las grandes corporaciones globales.

El capítulo más extenso se centra en evaluar los impactos que Iberdrola estaría generando según testimonios de diversos sectores afectados, destacándose entre otros la mercantilización de servicios públicos, la pérdida de soberanía, la practica del lobby, la falta de transparencia, la corrupción, la criminalización social, y la vulneración de derechos laborales, de comunidades y de pueblos indígenas. Para la identificación de los diferentes impactos el trabajo de campo ha sido trascendental ya que apenas se disponía de información e investigaciones realizadas por otros expertos. Las entrevistas realizadas a sectores afectados por la empresa han sido clave para poder evidenciar las prácticas poco adecuadas por parte de la corporación. Por una parte, hay que destacar los testimonios aportados por expertos del sector eléctrico mexicano (académicos, ingenieros). Por otra parte, es muy relevante la información obtenida de fuentes sindicales (directivos de sindicato). Finalmente, han sido muy valiosos los testimonios de primera mano aportados por la población afectada en aquellos lugares donde la empresa tiene proyectos de generación eléctrica, concretamente en el Estado de Oaxaca y más específicamente en el Istmo de Tehuantepec (campesinos, indígenas, vecinos de la comunidad)

\section{El sector eléctrico en México y la presencia de Iberdrola}

La llegada de Iberdrola y de otras multinacionales a México hay que situarla dentro del contexto de cambio de modelo de desarrollo que se implanta en el país, con la imposición de las recetas de corte neoliberal. El nuevo modelo, exigía una reducción sustancial del papel del Estado en el ámbito económico, lo que supuso la puesta en marcha de un proceso de privatizaciones intenso (TPP, 2014: 8). A partir de 1984 se reduce el número de empresas estatales de manera drástica, pasando de las 1.155 que había en 1982 a las 210 que quedarán en el año 2003. Se privatizaron sectores estratégicos como la siderurgia estatal, Teléfonos de México, los ferrocarriles, aeropuertos, etc. (Verbanaz, 2010: 8-9).

La privatización masiva está íntimamente relacionada con la apertura a la Inversión Extranjera Directa (IED), ya que una gran parte de los sectores estratégicos pasaron a manos de empresas foráneas (TPP, 2014: 8). En cuanto al origen del capital invertido, el principal país será EE.UU., seguido del Estado Español. En el año 2008, por 
ejemplo, el primero representó el $40,7 \%$ del total de la IED, mientras que el segundo supuso más de un 19\% (Verbanaz, 2010: 6)

La entrada de multinacionales españolas se produjo con mucha fuerza, fundamentalmente entre 1994 y 1999, donde la media anual de IED fue de 9.700 millones de dólares. El principal destino de la inversión fue el sector financiero, que representó casi el 50\% del total de la IED española en el país en el periodo 1993-2008, destacándose el BBVA y el Banco Santander. El siguiente sector en importancia fue el de suministro de energía eléctrica y gas, "siendo Iberdrola el protagonista en este ramo" (Verbanaz, 2010: 10).

Si nos centramos en el sector eléctrico, la ola neoliberal también se impuso con la reforma de la Ley del Servicio Público de Energía Eléctrica (LSPEE) en 1992, que permitió la entrada del capital privado en generación. Tras casi 20 años de expansión progresiva de las multinacionales, para fines de 2010 estas generaban el 33\% de la electricidad total del país (Hernández Ayala, 2010: 7).

En cuanto a la procedencia del capital, los principales inversores en el sector han sido multinacionales del Estado español (Iberdrola y Unión Fenosa), Estados Unidos (Intergen y AES), Canadá (Transalta) y Japón (Mitsubishi). Las de origen español (Iberdrola y Unión Fenosa) son las más importantes, ya que concentran el $70 \%$ de la producción privada: $8.413 \mathrm{MW}$ de un total de $11.907 \mathrm{MW}$ de capacidad de generación privada (Hernández Ayala, 2010: 7-12).

Iberdrola, por su parte, afirma que es el "primer productor privado de Electricidad en México, con 5.172 MW de potencia instalada" y, el segundo del país tras la estatal Comisión Federal de Electricidad (CFE) (Iberdrola, 2011c: 38). A finales de 2011 disponía de cinco centrales de ciclo combinado de gas y una de cogeneración, con una capacidad instalada de $4.968 \mathrm{MW}$, que suponían el $96 \%$ de su capacidad total de generación, frente a los $204 \mathrm{MW}$, que suponen el $4 \%$ restante, correspondientes a los tres parques eólicos instalados en el sur del país. Respecto a las plantas de ciclo combinado de gas, la primera que se puso en marcha fue la de Monterrey, en el año 2002, con una capacidad de 1.040 MW. Al año siguiente se activó la planta de Altamira, en el Estado de Tamaulipas, con una capacidad de $1.036 \mathrm{MW}$, mientras que en 2005 se abrió la central de La Laguna en Durango, de 516 MW. En 2006 se pone a funcionar Altamira V (Tamaulipas), con una capacidad de 1.121 MW y el siguiente año se pone en marcha la planta de Tamazunchale en San Luís Potosí, con 1.135 MW (Iberdrola, 2012a: 16). La central de cogeneración Enertek, también en Tamaulipas, dispone de $120 \mathrm{MW}$ y fue comprada por Iberdrola en el año 2001.

En cuanto a los parques eólicos, son proyectos más recientes: el parque de La Ventosa fue inaugurado en el año 2007, el Bi Nee Stipa fue comprado en enero de 2011 y La Venta III fue finalizado en el año 2012. Los tres se encuentran en el sur del país, en el Estado de Oaxaca, concretamente en el istmo de Tehuantepec, territorio donde desarrollamos nuestro trabajo de campo.

\section{Las grandes corporaciones y sus impactos}

La literatura crítica relativa a las grandes corporaciones y a los impactos que generan es extensa y se remonta muy atrás en el tiempo, desde el surgimiento y expansión del fenómeno de las empresas multinacionales (Teitelbaum, 2010: 36). A principios del nuevo siglo, en el marco del proceso de globalización neoliberal, diversos autores coinciden en identificarlas como una de las instituciones dominantes de nues- 
tro tiempo (Petras y Veltmeyer, 2007: 63; Achbar y Abbot, 2009; Sánchez Pérez, 2008; Zabalo, 2007: 7). Los impactos que generan son múltiples: económicos, sociales, ambientales...A continuación, vamos a señalar algunos de los impactos más habituales.

En el ámbito económico los impactos que generan las corporaciones son diversos. Por un lado, tenemos la mercantilización de servicios públicos (gas, electricidad, agua) que se ha producido en las tres últimas décadas, provocando la conversión de "bienes públicos" en "mercancías", y por tanto, tornando prioritaria la "lógica de lucro" frente a la "de servicio público" (Uharte, 2005: 110). Por otro lado, la reducción de soberanía nacional, cuando se permite la inserción extranjera en sectores estratégicos (Petras y Veltmeyer, 2007: 223). A su vez, debemos señalar los elevados márgenes de rentabilidad que obtienen, como consecuencia de la trasferencia de riqueza de manos públicas a privadas a través de diversos mecanismos: privatización a bajo costo, legislación que permite altos precios del servicio, subvenciones públicas y ventajas fiscales (reducción de impuestos), imposición de salarios bajos, etc.

En el terreno político, algunas corporaciones distorsionan el funcionamiento de la democracia, sirviéndose de diferentes instrumentos como la práctica del lobby'. Hernández Zubizarreta (2009b: 132) advierte de los "múltiples recursos" que las corporaciones invierten para influir en las decisiones políticas de organismos nacionales e internacionales. También destaca la corrupción a través del soborno a funcionarios, cargos gubernamentales, etc., a los que se les promete una remuneración a cambio de legislar a su favor, adjudicar contratos de obra o servicio... (Carrión y Verger, 2007: 12) $)^{2}$.

Esto evidencia que diferentes agentes son responsables de los impactos políticos que se producen. Por un lado, obviamente están las empresas, pero a su vez, como se señala en la sentencia del Tribunal Permanente de los Pueblos de 2010, también son corresponsables los Estados de donde proceden dichas empresas, los Estados que las reciben y permiten sus prácticas y los Tratados de Libre Comercio que amparan estas actuaciones (TPP, 2010: 26-27).

El nuevo marco jurídico favorable a las multinacionales que se ha ido configurando a nivel internacional es otro aspecto clave. La asimetría entre la evolución del derecho comercial global y la del derecho internacional de los derechos humanos es cada vez mayor (Hernández Zubizarreta, 2009a: 20-3; Hernández Zubizarreta y Ramiro, 2015). La criminalización de grupos opositores es otro impacto reseñable. Ramiro (2011: 11) apunta que "en muchos casos, los integrantes de organizaciones sociales que han criticado el poder de las corporaciones han sido criminalizados y reprimidos por la fuerza pública o por paramilitares".

Los diversos impactos ambientales constituyen otra dimensión relevante. Destacan las múltiples formas de contaminación (emisión de gases efecto invernadero, ver-

1 En el informe del PNUD de 2004 sobre "La democracia en América Latina" algunos presidentes de la región destacaron la influencia creciente de los lobbys de las grandes corporaciones y reconocieron que esto suponía un "obstáculo a la democracia" (Hernández Zubizarreta, 2009b: 118).

2 Al ser una práctica "oculta" resulta difícil de medir, aunque la OCDE (Organización para la Cooperación y el Desarrollo Económico) calcula que el soborno internacional se acerca los 150.000 millones de dólares al año en todo el mundo, y Transparencia Internacional lo eleva a los 400.000 millones (Hernández Zubizarreta, 2009b: 128-9). 
tidos tóxicos...), y la destrucción del hábitat y la biodiversidad. La presencia de las corporaciones en áreas muy contaminantes como las industrias extractivas (hidrocarburos, minería...) las coloca en una posición de primer orden entre los agentes responsables (Petras y Veltmeyer, 2007: 228).

En el plano laboral los impactos son también diversos, tanto en relación con el derecho al trabajo, como con el derecho a trabajar en condiciones dignas Reducción salarial, fin de la estabilidad laboral, supresión de convenios colectivos, subcontratación, inseguridad laboral, discriminación por género y etnia, son algunas de las principales denuncias que reciben (Teitelbaum, 2010: 105). A esto hay que añadir el ataque a los derechos sindicales como la libertad de afiliación, el respeto a la acción sindical sin represalias, etc. (De la Fuente et al, 2013).

Las comunidades de los lugares donde las corporaciones tienen presencia se convierten también en sectores afectados. Los impactos son diversos según el tipo de empresa: desplazamientos de población, pérdida de tierras, impactos en la economía local (agricultura, pesca...), deterioro del tejido social. También destacan los impactos que padecen las y los usuarios de servicios públicos (distribución de gas, agua, electricidad): aumentos desproporcionados de tarifas, exclusión de grupos de población de barrios populares o de zonas rurales no consideradas "rentables" (Ramiro, 2011: 10-11).

Los pueblos indígenas son también un grupo especialmente vulnerable a las prácticas de las corporaciones. Las agresiones a su territorio - tanto a su propiedad como a sus recursos - y a su identidad cultural son algunas de las violaciones más denunciadas. Berraondo (2008: 93) asegura que existe un "cuadro recurrente" de violaciones como la expropiación de tierras, la reubicación, la inundación de sus territorios, la destrucción medioambiental de su hábitat, la represión, etc. (Ramiro y González, 2010: 189-200).

\section{Los impactos de Iberdrola en México}

Soberanía. La privatización progresiva de la generación de electricidad en México, que se produce en 1992 como consecuencia del cambio normativo en la Ley del Servicio Público de Energía Eléctrica (LSPEE), ha permitido la entrada de multinacionales y esto ha debilitado la soberanía energética del país según el investigador Hernández Ayala (2012).

Teniendo en cuenta los datos aportados por Hernández Ayala la totalidad de capacidad de generación de producción privada (11.907 MW para 2009), está en manos de corporaciones extranjeras. Iberdrola sería la primera con $4.239 \mathrm{MW}$ (un 35\% del total). Le seguiría la también española Gas Natural/Unión Fenosa con 4.174 MW (otro 35\%). Mitsubishi (Japón) tenía para esa fecha 1.515 megavatios de capacidad instalada (12\%), mientras que Intergen de EE.UU., 984 MW (8\%). Finalmente destacan la canadiense Transalta, con $511 \mathrm{MW}(4 \%)$ y la estadounidense AES con 484 MW (4\%) (Hernández Ayala, 2010: 7).

Los datos muestran una la tendencia creciente, ya que de 2001 a 2009, las multinacionales aumentaron su capacidad de generación del $1,3 \%$ al $22,7 \%$, mientras que la del Estado se redujo del $98 \%$ al $77 \%$. En términos de producción real las corporaciones pasaron de representar el $0,62 \%$ en 2001 al $33,16 \%$ en 2009 , lo que supuso una reducción drástica de la producción estatal, del $99 \%$ al $66 \%$ como se muestra en las siguientes tablas. 
Tabla 1. Capacidad en MW de las multinacionales y de la Comisión Federal de Electricidad

\begin{tabular}{|c|c|c|c|c|c|c|c|c|}
\hline & 2001 & 2003 & 2004 & 2005 & 2006 & 2007 & 2008 & 2009 \\
\hline CFE & 36.236 & 36.971 & 38.422 & 37.325 & 37.470 & 38.397 & 38.474 & 38.927 \\
\hline Mult. & 484 & 6.756 & 7.265 & 8.251 & 10.387 & 11.457 & 11.457 & 11.457 \\
\hline $\begin{array}{c}\text { Total } \\
\%\end{array}$ & 36.720 & 43.727 & 45.687 & 45.576 & 47.857 & 49.854 & 49.931 & 50.384 \\
\hline $\begin{array}{c}\text { Mult } \\
\%\end{array}$ & $1,36 \%$ & $15,45 \%$ & $15,9 \%$ & $18,1 \%$ & $21,7 \%$ & $23 \%$ & $22,94 \%$ & $22,73 \%$ \\
\hline \begin{tabular}{c} 
CFE \\
\hline
\end{tabular} & $98,64 \%$ & $84,55 \%$ & $84,1 \%$ & $81,9 \%$ & $78,3 \%$ & $77 \%$ & $76,06 \%$ & $76,27 \%$ \\
\hline
\end{tabular}

Fuente: Hernández Ayala (2010: 7)

Tabla 2. Produccion en TWH de las multinacionales y de la Comisión Federal de Electricidad

\begin{tabular}{|c|c|c|c|c|c|c|c|}
\hline & 2001 & 2003 & 2005 & 2006 & 2007 & 2008 & 2009 \\
\hline CFE & 190.88 & 169.32 & 170.07 & 162.47 & 157.51 & 157.16 & 154.14 \\
\hline Mult & 1.20 & 31.62 & 45.56 & 59.43 & 70.98 & 74.23 & 76.50 \\
\hline Totall & 192.08 & 200.94 & 215.63 & 221.90 & 228.49 & 231.40 & 230.64 \\
\hline$\%$ CFE & $99,38 \%$ & $84,27 \%$ & $78,88 \%$ & $74,22 \%$ & $69,94 \%$ & $67,93 \%$ & $66,84 \%$ \\
\hline$\%$ Mult & $0,62 \%$ & $15,73 \%$ & $21,12 \%$ & $26,78 \%$ & $31,06 \%$ & $32,07 \%$ & $33,16 \%$ \\
\hline
\end{tabular}

Fuente: Hernández Ayala (2010: 7)

Mercantilización. La privatización de la generación eléctrica ha estimulado un proceso de mercantilización de un servicio básico, afectando directamente la capacidad de consumo de una parte importante de la población según Obrador, ingeniero de la CFE (2012). La privatización se impulsó por la necesidad teórica de aumentar la capacidad de producción en función de la demanda creciente que iba a tener el país y porque las plantas del Estado no iban a poder, por sí solas, generar toda la electricidad necesaria, agrega Obrador. Sin embargo, Obrador considera que fue "una decisión política más que técnica": "hubo un boom de construcción de plantas eléctricas de 2000 a 2006, de productores externos que no requeríamos; se le dio preferencia a los productores externos y la nacional fue decreciendo (...) se fueron poniendo en reserva fría las plantas estatales" (Obrador, 2012). Hernández Ayala (2010: 7) precisa que la CFE redujo su volumen de producción de 190,88 a 154,14 TWh, es decir, en casi un $20 \%$.

Según criterio de Israel Rodríguez (2011), experto en el sector energético mexicano, la tendencia continuará ya que, el "gobierno mexicano a través de la CFE tiene programado el retiro de 11.093 megavatios de capacidad del servicio público durante los próximos 15 años", según establece la "nueva prospectiva del sector eléctrico 20102025, elaborada por la Secretaría de Energía (Sener)”.

Rentabilidad. El negocio eléctrico le genera a Iberdrola buena rentabilidad según los informes de la propia empresa. El Ebitda ("beneficio antes de intereses, impuestos, depreciaciones y amortizaciones") global de lberdrola de 2010 aumentó en comparación con el año anterior un 10,5\%. En México el crecimiento fue superior a la media, un $13,8 \%$, según datos de la propia Iberdrola (2011d: 160).

Eficiencia. Diversos investigadores mexicanos consideran que la entrada de multinacionales en la generación de electricidad ha provocado problemas de ineficiencia en la gestión pública. El primer foco de ineficiencia se habría generado por la notable reducción de producción del sector público y la excesiva capacidad ociosa. A escala internacional, según los expertos, la mayoría de los países tienen alrededor de un $20 \%$ de capacidad ociosa en la generación eléctrica, es decir, un número de plantas 
que no producen pero que podrían ponerse en funcionamiento si fuera necesario en casos de emergencia. En México, este porcentaje se duplica, llegando a un $40 \%$ de capacidad ociosa, según datos de Hernández Ayala (2010).

Según Garduño (2012) esta decisión evidencia un manejo ineficiente del sector, ya que el Estado debe dedicar un monto de capital a mantener plantas que no producen, y además tiene que desembolsar un importante volumen de dinero para comprar a las privadas, mientras que antes lo producía él mismo. Garduño indica que en el ejercicio 2010 la Comisión Federal de Electricidad (CFE) "pagó a empresas privadas generadoras de energía 74 mil 245 millones de pesos", lo que representa el "30\% del presupuesto total de la empresa".

Otro foco de ineficiencia sería a causa de la sustitución de plantas estatales que producen a un precio más barato (hidroeléctricas, carbón), por centrales de ciclo combinado de gas privadas que generan electricidad a un coste mayor, según consideran diversos expertos (Obrador, 2012; Martínez, 2012). Iberdrola sería un exponente de esto ya que el $96 \%$ de sus plantas de generación son de ciclo combinado, es decir, de producción más cara.

Lobby. Según el Sindicato Mexicano de Electricistas, "es difícil precisar el grado de complicidad de la clase política mexicana con las transnacionales hispanas" (SME, 2010). Lilia (2009), investigadora del sector energético, destaca las declaraciones del presidente de la Comisión de Puntos Constitucionales del Senado, Manuel Barttlet, quien le manifestó en una de sus investigaciones que "en el Legislativo las transnacionales cabildeaban directamente la privatización del sector eléctrico, que el Partido Acción Nacional disfrazó como reforma estructural".

El Sindicato Mexicano de Electricistas (SME) por su parte, señala que las multinacionales eléctricas españolas se apoyaron en el lobby vinculado al "despacho jurídico del ex senador Diego Fernández, al cual pertenece el actual Secretario de Gobernación de México" (SME, 2010).

Corrupción. En los proyectos eólicos que Iberdrola tiene en Oaxaca, al sur del país, los afectados entrevistados indican que la empresa sobornó a las autoridades municipales, ya que estas intentaron convencerles de alquilar sus tierras para instalar los molinos (Valencia, 2012; López, 2012). Otro denuncia de corrupción está relacionada con las subcontratas. Santiago Artea (2012), quien ha trabajado en diversas obras de construcción de parques de Iberdrola, entre ellos, el de La Venta III en Oaxaca, señala que cuando la empresa para la que trabajaba intentó concursar para un contrato de servicios, en la sede de Iberdrola de México les dijeron que si querían trabajar tenían que pagar un soborno: "en México DF nos dijeron que teníamos que dar el diezmo para poder trabajar (...) nos lo dijeron en las oficinas de Iberdrola de México"3.

llegalidad. Leticia Campos, doctora de la Universidad Autónoma de México y experta en el sector energético mexicano indica que la reforma de la Ley del Servicio Público de Energía Eléctrica (LSPEE) en 1992, tuvo como objetivo de permitir la entrada del sector privado. Esta reforma ha sido considerada por diversos expertos como un acto inconstitucional y por tanto ilegal, ya que una ley de menor rango no puede contradecir a la ley máxima del país, la Constitución (Campos, 2012; Hernández Ayala, 2010; Montes de Oca, 2012). Según el artículo 27 de la Constitución, "corresponde exclusivamente a la Nación generar, conducir, transformar, distribuir y

3 "El diezmo" aclara Artea (2012), "es una cantidad de dinero que hay que darle a un representante de la empresa para que la quieres trabajar, si quieres conseguir algún tipo de contrato con ellos". Esta persona recibe un dinero y te garantiza que luego te contratarán. 
abastecer energía eléctrica que tenga por objeto la prestación de servicio público" y que "en esta materia no se otorgarán concesiones a los particulares".

Transparencia. La supuesta falta de transparencia en relación a la instalación de los proyectos eólicos de Iberdrola en Oaxaca ha sido un aspecto que han destacado diversos sectores afectados. Bettina Cruz, líder comunitaria, indica que Iberdrola prometió a los campesinos "mucho dinero" si firmaban los contratos de arrendamiento. Les hicieron "firmar contratos leoninos" aprovechándose en muchos casos del "desconocimiento normativo de muchos campesinos". Lo hicieron de "manera separada para que no pudieran organizarse y negociar colectivamente por un precio más alto" (Cruz, 2012). Otro indicador relevante, según la Asamblea de los Pueblos del Istmo en Defensa de la Tierra $(2011 b)^{4}$, es que tras la firma de los contratos, estos no fueron entregados a los ejidatarios hasta dos años después: "a dos años de haber firmado el contrato con Iberdrola, ésta no nos ha proporcionado copia, violando así nuestros derechos".

Criminalización. En los parques eólicos de Iberdrola en Oaxaca la Asamblea de los Pueblos del Istmo en Defensa de la Tierra (2011a) tiene registradas diversas denuncias contra "el representante de Iberdrola" por haber amedrentado a los ejidatarios del parque La Venta III. En el parque de La Ventosa, también gestionado por Iberdrola, los testimonios de habitantes del municipio son relevantes. López Toledo (2012), ejidatario de la zona que alquiló terrenos a la multinacional, indica: "me amenazaron de muerte (...) que tenía que estarme quieto (...) hasta con mi familia me amenazaron". Bettina Cruz (2012), afirma que cuando los opositores se han atrevido a manifestarse realizar paros y bloqueos, las "empresas han enviado a la policía para que los reprimiera" 5 . Estos testimonios reflejarían un incumplimiento de Iberdrola de su Código Ético.

Ambiental. Si revisamos los datos que hemos presentado anteriormente, para fines de 2011 las cinco centrales de ciclo combinado de gas y la de cogeneración suponían el $96 \%$ de su capacidad total, mientras que el $4 \%$ restante correspondía a tres parques eólicos instalados en Oaxaca. Esto significa que el $96 \%$ de la energía producida en el país es no es limpia porque las plantas de ciclo combinado de gas coadyuvan al cambio climático: emiten $\mathrm{CO}_{2}$, principal responsable del cambio climático, cantidades significativas de metano y óxido de nitrógeno ${ }^{6}$. Además, requieren un consumo de agua desproporcionado y reducen la calidad de esta porque "utilizan productos fitotóxicos para limpiar las conducciones de agua” (Ecologistas en Acción, 2004).

En cuanto a los proyectos a futuro de Iberdrola en México, dos de ellos no son de producción limpia ni segura. Por un lado, tenemos la adjudicación en 2011 de la construcción y puesta en marcha de la planta de cogeneración de 430 megavatios en el Estado de Guanajuato, en las instalaciones de PEMEX (Petróleos Mexicanos) (EI

4 Algunos ejidatarios también se quejan de que los representantes de Iberdrola hicieron desde el principio muchísimas promesas sobre puestos de trabajo que se iban a crear y grandes beneficios que iba a obtener el municipio, pero nunca se ha cumplido nada. López Toledo (2012), ejidatario de La Ventosa, asegura que "cuando llegó el representante de lberdrola ( ) ese cuate hizo muchas promesas ( ) que iban a poner una fábrica de palas en el municipio ( ) hablaba de miles de obreros que iban a trabajar haciendo palas para las molinos".

5 La propia Bettina Cruz ha sido una de las personas más reprimidas en el conflicto con Iberdrola y con el resto de las transnacionales eólicas. Debido a las amenazas de muerte que recibió tuvo que salir del municipio y esconderse durante mes y medio (Cruz, 2012).

6 "Los óxidos de nitrógeno bajo la acción de la luz solar, sobre todo en verano, provocan la formación de ozono" (Ecologistas en Acción, 2004). 
Mundo, 2011). El combustible de esta planta será gas, por lo que se producirán impactos ambientales debido a las emisiones de $\mathrm{CO}_{2}$ y de óxido de nitrógeno. Por otro lado, Iberdrola asumió la gestión desde 2011 de la central nuclear "Laguna Verde" en el Estado de Veracruz, según Obrador (2012). Un emprendimiento de alto riesgo por su componente nuclear.

Laboral. Iberdrola en su "Código Ético" (Iberdrola, 2011b: 5-6) y en su "Política General de Responsabilidad Social Corporativa" (Iberdrola, 2011a) asegura que garantiza unas buenas condiciones laborales a todas y todos sus empleados. Algunos testimonios recopilados en nuestra investigación muestran una realidad diferente.

En los proyectos eólicos que tiene Iberdrola en Oaxaca la empresa ha priorizado el trabajo a través de subcontratas, lo cual ha precarizado las condiciones laborales de sus operarios, según testimonios de los propios trabajadores. López Toledo (2012), trabajador de un proyecto eólico, indica que en "La Ventosa" Iberdrola "no les contrató directamente sino que la empresa contrata a subcontratas para pagar menos a sus trabajadores (...) la gente, como no hay trabajo, a fuerza tienen que aceptar". Por otro lado, los testimonios muestran vulneraciones del principio de la libertad sindical. En los parques de' La Venta III', Valencia (2012), trabajador eólico, afirma que "la mafia de la SUTERM es la que vende el trabajo (...) si quieres trabajar les tienes que pagar primero (...) ellos se llevan un porcentaje".

Usuarios. En México en la última década se ha producido un aumento constante y sustancial del precio de la luz. Según el investigador José Luis Apodaca, del Observatorio Ciudadano de la Energía, entre los años 2000 y 2008 se produjo un incremento medio del 64\% en la tarifa doméstica (citado en Hernández Ayala, 2010: 15). Esto ha provocado el surgimiento de organizaciones de usuarios contra las altas tarifas: la Red Nacional de Resistencia contra las Altas Tarifas de la Energía Eléctrica, en el norte y sur del país y, la Asamblea Nacional de Usuarios de Energía Eléctrica, en la capital y en los Estados del centro de México.

La responsabilidad de las privadas extranjeras sería indirecta según diversos expertos. Obrador (2012), ingeniero de la Comisión Federal de Electricidad (CFE) indica que los productores privados como Iberdrola venden más caro a la CFE y esta, en consecuencia, le aplica entonces el incremento a los y las ciudadanas. Lilia (2009), experta en temas energéticos, recuerda que la Auditoría Superior de la Federación (ASF) detectó el indiscriminado incremento de tarifas. La Asamblea Nacional de Usuarios de Energía Eléctrica (2012) no sólo responsabiliza al Estado del aumento de las tarifas sino que lo vincula al proceso de privatización y a la entrada de corporaciones ${ }^{7}$.

Comunidad. Los testimonios recogidos en el trabajo de campo muestran que los proyectos eólicos de Iberdrola en Oaxaca, estarían provocando impactos socio-comunitarios. Por un lado en La Ventosa, López Toledo (2012), habitante de la comunidad, apunta que Iberdrola "ha conseguido enfrentar a los campesinos entre sí, ofreciendo dinero para que se desvincularan de las movilizaciones". Por otro lado, en Juchitán de Zaragoza, el núcleo urbano más grande cercano a varios parques eólicos, Bettina Cruz (2012) señala impactos de género, "con el aumento exponencial

7 La resistencia ciudadana contra las altas tarifas está siendo duramente reprimida, según manifiestan en entrevista varias personas pertenecientes a la Asamblea Nacional de Usuarios de Energía Eléctrica (2012). El alto precio de la luz ha llevado a impulsar iniciativas en los barrios más pobres para no pagar los recibos, lo que ha traído como consecuencia la criminalización, detención de líderes y amenaza de embargos. 
de la prostitución asociado a la llegada de trabajadores y de personal extranjero". El "aumento de precios de la vivienda y de los alimentos" es otro impacto asociado a la llegada de los emprendimientos eólicos, según Artea (2012) ${ }^{8}$, habitante de Santo Domingo.

Pueblos indígenas. Iberdrola en su "Código Ético" se "compromete a respetar los derechos de las minorías étnicas y de los pueblos indígenas en los lugares donde desarrolle su actividad" (Iberdrola, 2011b: 5). Sin embargo, se han producido conflictos con algunos grupos indígenas según señala la líder indígena Bettina Cruz. Para Cruz, se está produciendo un impacto cultural por el choque de imaginarios en torno a la concepción del viento, ya que las multinacionales conciben al viento como un recurso más que se puede convertir en mercancía y por tanto ser fuente de lucro, mientras que para algunos grupos de los pueblos originarios ${ }^{9}$ es parte de un elemento vital y sagrado, que no puede estar sujeto a la lógica del capital: "Para nosotros el viento es uno de los elementos de la vida; en zapoteco reconocemos el viento del norte, el viento del sur, el viento del agua (...) tienen nombres diferentes (...) para los pueblos ikoots los vientos son deidades, son su dioses y diosas (...) el viento mujer es la brisa (...) para nosotros tiene un significado diferente al que tienen las empresas (...) nosotros no habíamos visto el viento como mercancía".

\section{Conclusiones}

Iberdrola se ha convertido en una de las multinacionales que mayor penetración han tenido durante el proceso de privatización del sector eléctrico en México. Los beneficios de la empresa han sido destacables durante la época de la crisis económica y en el caso de México estos han estado por encima de la media. Esto se ha producido a la vez que las tarifas eléctricas en el país han sufrido un aumento constante y sustancial. Teniendo en cuenta que México es un territorio sumamente lucrativo para la multinacional, parece claro que el país continuará siendo un lugar estratégico para los negocios de la corporación.

La red de alianzas que ha ido tejiendo en México, además de su capacidad de incidencia en las autoridades políticas, como lo indican los testimonios recogidos en el trabajo de campo realizado en Oaxaca, muestran un poder creciente de los grandes agentes económicos. El supuesto trato de favor que algunos gobiernos municipales del Istmo de Tehuantepec, donde Iberdrola tiene negocios eólicos, están brindando a la empresa, serían un indicador relevante en este sentido. Todo esto debilita las aspiraciones de fortalecer los regímenes democráticos y muestra una injerencia excesiva de los poderes económicos en la vida política.

La imagen de empresa 'verde' que impulsa proyectos de energía renovable y sostenible y que respeta los derechos laborales y los de los pueblos indígenas no se corresponde con la realidad según los testimonios recogidos. Los fuertes conflictos sociales que se han generado en Oaxaca, alrededor de sus parques eólicos, rompen el principio de sustentabilidad que Iberdrola teóricamente promociona. Además, estos conflictos se están desarrollando en un territorio muy complejo y diverso como

8 Santiago Artea habitante de Santo Domingo Ingenio se queja de que "aquí la vida es más cara que en otros municipios ( ) ha habido una inflación por todos estos proyectos ( ) antes rentabas una casa por 500 pesos y ahorita no baja de 1.500 ( ) el kilo de carne estaba a 40 y ahora está a 80 ( ) todo subió pero el salario es el mismo".

9 Se calcula que un $32 \%$ de los habitantes de Oaxaca, es decir, un tercio de la población, pertenece a pueblos indígenas (Sipaz, 2012) 
el Istmo de Tehuantepec, con fuerte sustrato indígena, lo cual está provocando el surgimiento de un discurso contrario a las empresas españolas, a las cuales algunos grupos comunitarios acusan de colonizadoras. En este contexto, es muy probable que los niveles de conflictividad continúen muy altos en el corto plazo.

\section{Fuentes}

Achbar, M. Abbot, J. (2009). La Corporación. Documental. ISAAN Entertainment S.L.

Artea, S. (2012). Entrevista personal. 29-03-2012. Oaxaca.

Asamblea de los pueblos del istmo en defensa de la tierra y el territorio (2011a). "Ejidatarios del polígono La Venta III. Santo Domingo Ingenio, Oaxaca. Estragos causados por el parque eólico La Venta III de Iberdrola se han quintuplicado". oaxacalibre.org. 14-05-2011

http://oaxacalibre.org/index.php?option=com_content\&view=article\&id=2997: apo yo-y-la-solidaridad-en-su-lucha-pide-la-asamblea-de-los-pueblos-del-istmo\&catid=21

Asamblea de los pueblos del istmo en defensa de la tierra y el territorio (2011b). " ${ }^{\circ}$ Encuentro de nuestras voces de lucha y resistencia". Tierra y Territorio. 24-07-2011. http://oaxacalibre.org/index.php?option=com_content\&view=article\&id=2997: apo yo-y-la-solidaridad-en-su-lucha-pide-la-asamblea-de-los-pueblos-del-istmo\&catid=21

Asamblea Nacional de usuarios de la energía eléctrica (2012). Entrevista grupal. 19-032012. México D.F.

Berraondo, M. (2008). "Aplicación del derecho o programas de RSC para pueblos indígenas". Revista de Relaciones Laborales "Lan Harremanak", n 19, 89-116.

Campos, L. (2012). Entrevista personal. 22-03-2012. México D.F.

Carrión, J., Verger, T. (2007). “Amistades peligrosas: empresas transnacionales, poder político y poder mediático". En AA.VV. Las empresas transnacionales en la globalización. Madrid: OMAL.

Cruz, B. (2012). Entrevista personal. 26-03-2012. Oaxaca.

De la Fuente, M. et al (2013). Empresas transnacionales en América Latina. Análisis y propuestas del movimiento social y sindical. Bilbao: UPV-Hegoa.

Ecologistas en Acción (2004). "Campaña contra la instalación de centrales térmicas". ecologistasenacción.es, noviembre 2004.

http://ecologistasenaccion.es/article1191.html

El Mundo (2011). "México adjudicará a Iberdrola un proyecto de 230 millones de dólares.

El Mundo, 28-02-2011. Madrid.

http://www.elmundo.es/america/2011/02/28/mexico/1298908620.html

Garduño, R. (2012). "La Comisión pagó más de $\$ 74$ mil millones a la IP por generación de luz". La Jornada, 19-02-2012. México.

Hernández Ayala, J. (2010). "El papel del outsorcing en el proceso de privatización del servicio público de energía eléctrica en México". Manuscrito facilitado por el autor. México.

Hernández Ayala, J. (2012). Entrevista personal. 22-03-2012. México DF. 
Hernandez Zubizarreta, J. (2009a). "¿Lex mercatoria o derechos humanos? Los sistemas de control de las empresas transnacionales". En J. Hernández Zubizarreta y P. Ramiro (eds.). El negocio de la responsabilidad. Crítica de la Responsabilidad Social Corporativa de las empresas transnacionales. Barcelona: Icaria\&Antrazyt.

Hernández Zubizarreta, J. (2009b). Las empresas transnacionales frente a los derechos humanos: historia de una asimetría normativa. Bilbao: Hegoa.

Hernandez Zubizarreta, J. y Ramiro, P. (2015). Contra la lex mercatoria. Propuestas y alternativas para desmantelar el poder de empresas transnacionales. Barcelona: Icaria.

IBERDROLA (2011a). Política general de Responsabilidad Social Corporativa. IBERDROLA.

http://www.iberdrola.es/webibd/gc/prod/es/doc/responsabilidad_rsc.pdf

IBERDROLA (2011b). Código ético. IBERDROLA.

https://www.iberdrola.es/webibd/gc/prod/es/doc/normascodigoiberdrola.pdf

IBERDROLA (2011c). Informe anual 2010. IBERDROLA.

IBERDROLA (2011d). Cuentas consolidadas 2010. IBERDROLA.

IBERDROLA (2012a). Informe de resultados. Nueve meses de 2011. IBERDROLA.

Lilia, A. (2009). "Privatización de facto del sector eléctrico". Revista Fortuna. 15-10-2009.

López, O. (2012). Entrevista personal. 29-03-2012. Oaxaca.

López Toledo, C. (2012). Entrevista personal. 27-03-2012. Oaxaca.

Martínez, J. (2012). Entrevista personal. 23-03-2012. México D.F.

Montes de Oca, J. (2012). Entrevista personal. 19-03-2012. México. DF.

Obrador, J. (2012). Entrevista personal. 31-03-2012. México D.F.

Rodriguez, I. (2011). "En 2010 la CFE pagó 15 mil mdd a extranjeros por electricidad”. La Jornada. 22-04-2011. México.

Petras, J., Veltmeyer, H. (2007). Juicio a las multinacionales. Inversión extranjera e imperialismo. Madrid: Editorial Popular.

Ramiro, P., González, E. (2010). "Multinacionales españolas en América Latina: impactos sobre los pueblos indígenas". En A. Cruz et al. Pueblos originarios en América. Guía introductoria de su situación. Pamplona: ALDEA. pp. 189-200.

Ramiro, P. (2011). "El segundo desembarco: los impactos de las multinacionales españolas en América Latina”. En S. Álvarez (coord.). Convivir para perdurar. Conflictos ecosociales y sabidurías ecológicas. Barcelona: Icaria-Antrazyt. (Manuscrito facilitado por el autor antes de la publicación).

Sanchez Pérez, J. (2008). "El poder de las empresas multinacionales". X Coloquio Internacional de Geocrítica. Diez años de cambios en el mundo, en la geografía y en las Ciencias Sociales, 1999-2008. Barcelona 26-30 de mayo. Universidad de Barcelona.

SIPAZ (2012). Oaxaca en datos. México: SIPAZ.

SME (Sindicato Mexicano Electricistas) (2010). "Empresa transnacional a la que se acusa". Madrid: Tribunal Permanente de los Pueblos. 
Teitelbaum, A. (2010). La armadura del capitalismo. El poder de las sociedades transnacionales en el mundo contemporáneo. Barcelona: Icaria-Antrazyt.

TTP (2010). Tribunal Permanente de los Pueblos. La Unión Europea y las empresas transnacionales en América Latina. Universidad Complutense de Madrid. 14-17 de mayo.

TTP (2014). Tribunal Permanente de los Pueblos. Libre comercio, violencia, impunidad y derechos de los pueblos en México (2011-2014). Ciudad de México. 12-15 de noviembre.

Uharte, L. (2005). "Política social y democracia: un "nuevo" paradigma". Revista Venezolana de Economía y Ciencias Sociales, vol. 11, $n^{\circ} 3,93-114$.

Valencia, R. (2012). Entrevista personal. 28-03-2012. Oaxaca.

Verbanaz, S. (2010). "La inversión extranjera directa en el sector financiero mexicano". Informes OMAL. 3/2010. Madrid: OMAL.

http://www.omal.info/www/IMG/pdf/03INFORMESOMAL_s.verbanaz.pdf

Zabalo, P. (2007). "El papel de las multinacionales en la economía globalizada”. En AA.VV. Las empresas transnacionales en la globalización. Madrid: OMAL. 
